\title{
La publicación electrónica: ¿Un paradigma de organización documental digital?
}

\author{
RAMIRO LAFUENTE LÓPEZ \\ Centro Universitario de Investigaciones Bibliotecológicas \\ Tel: (525) 623-0355, FAX: (525)550-7461 \\ E-mail: lafuente@ servidor.unam.mx \\ ANgé liCA María Rosas GuTiÉrRez \\ Centro Universitario de Investigaciones Bibliotecológicas \\ Tel: (525) 623-03-68 FAX:(525)550-7461 E-mail: rosas@ cuib.unam.mx
}

\begin{abstract}
RESUMEN
La publicación electrónica se encuentra estrechamente relacionada con la tecnología que la hace posible, y ha introducido modificaciones en los procesos de edición de documentos, dando lugar a la creación de nuevas formas, procesos y medios de publicación que conducen a la creación de un paradigma de organización documental digital.

El lenguaje utilizado para aludir a la publicación electrónica deriva tanto del campo delas tecnologías delainformación, como del utilizado por las empresas dedicadas a la producción de software para publicación en línea y en cd-rom. Hasta el momento no existe en la literatura una claridad sobre los conceptos relacionados con este fenómeno. Por ello en el presente trabajo se identifican, sistematizan y estructuran aquellos elementos que se considera pueden permitir conceptuar el fenómeno de la publicación electrónica
\end{abstract}

\section{ELECTRONIC PUBLISHING: A PARADIGMFORDIGITAL DOCUMENT ORGANIZATION? \\ RAMIRO LAFUENTE-LÓPEZ ANGÉliCa MARÍA ROSAS-GUTIÉRREZ}

\begin{abstract}
Electronic publishing is closely related to the technology that makes it possible. Consequently, it is a phenomenon which poses for the electronic publishing environment in particular, and for publishing in general, modifications to the editorial process, especially with respect to the conception of the idea of a publishable product, as well changes in the concepts of publication, since in many cases it refers to an updating type of service.

The generation of the electronic publication thus requires the use of concepts derived both from the use of such technology and from the manufacturers of software for the publishing industry. Up to the present time there has been a lack of clarity and hierarchy in the literature in respect to the concepts related to this phenomenon. It is for this reason that the present study identifies, classifies, and structures the elements that conceptualize the electronic publication.
\end{abstract}

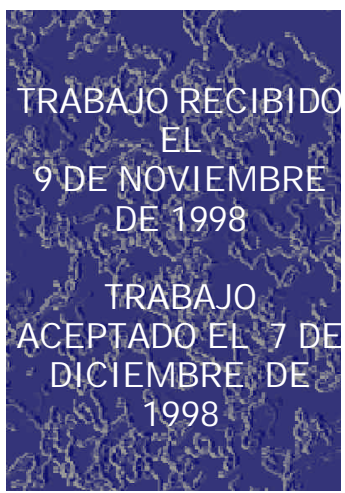




\section{INTRODUCCIÓN}

F lfenómeno dela publicación electrónica está estrechamente asociado a la socialización del uso de las computadoras, de las tecnologías de la información y al crecimiento de las redes de telecomunicación para transmitir información. Como no podemos separarlo de las tecnologías que la hacen posible, puesto que éstas determinan el perfil de manufactura de los documentos digitales, es indispensable identificar algunas de las tendencias predominantes en cuanto a las tecnologías aplicadas al diseño, producción y difusión de documentos digitales, a partir de las cuales se puedan inferir las características de esta nueva forma de organización documental para intercambiar y difundir información. ${ }^{1}$

La publicación electrónica está conformada esencialmente por texto digital, al cual lo consideramos como un conjunto de palabras transformadas a datos numéricos que son legibles por medio de una computadora, misma que deberá contener la tecnología necesaria para procesarlo, es decir, estructurarlo, almacenarlo, consultarlo, transmitirlo. Empero, el proceso de lectura-escritura de textos digitales no constituye por sí mismo el fenómeno de la publicación electrónica. En otros términos, una publicación electrónica puede estar constituida por un texto digital de cualquier tamaño, forma y naturaleza, pero esto no implica que todo texto digital sea una publicación electrónica. de:

La publicación electrónica es un fenómeno social que se caracteriza por el uso

* la automatización para el proceso de edición y difusión de textos digitales,

* programas para escribir, corregir, estructurar, consultar, leer y difundir textos digitales,

- las telecomunicaciones, o dispositivos electromagnéticos, para transmitir y difundir los textos digitales,

* convencionalismos sociales acerca de las características que debe cumplir una publicación electrónica en cuanto a su estructura formal, funcionamiento y cualidades del contenido temático,

* intenciones y finalidades que sustentan la organización de actividades encaminadas a fomentar la creación y difusión de textos digitales.

En términos generales podríamos afirmar que la idea de publicación electrónica busca construir nuevos medios, espacios y software para allanar el intercambio y difusión de información y con ello facilitar la generación de conocimientos. Aun cuando se ha hecho un mayor hincapié en el estudio y desarrollo de las estructuras que simplifiquen la publicación de documentos digitales, es indispensable el promover el estudio e investigación de la forma lógica que deben adoptar los contenidos temáticos dedicados a configurar un documento digital, a fin de especificar es-

1 Cf. Greg Anderson. "Virtual Q ualities for electronic publishing." En: Theuritual library. visiansand relities- Editado por Laverna M. Saunders. - Londres: Meckler, 1993.- p. 87- 110. 
tructuras lógicas y formales que ayuden a mejorar las cualidades de los mensajes que se trata de transmitir.

La tecnología para publicar un documento digital, le imprime al mismo diversas características en cuanto a su apariencia, organización y modo de uso. Tecnologías similares para la edición de un documento digital, originan publicaciones distintas, aun cuando ambas tengan elementos comunes entre sí. Por ejemplo, la tecnología adobe acrobat produce documentos digitales diferentes a la de hipertexto del html. Esta característica de la publicación electrónica la hace diferente de la imprenta, puesto que mientras la imprenta representa un solo tipo de propuesta técnica, en donde pueden variar las formas de producción pero no las características formales del producto (el impreso); en la publicación electrónica la aplicación de una tecnología específica genera un producto (documento digital) con características formales diferentes de los provenientes de otra tecnología.

La tecnología que ha logrado un mayor mercado para producir y publicar documentos digitales es aquella que se sustenta en la utilización del proceso de datos orientado a la realización de múltiples operaciones, en donde la producción de documentos digitales es una de ellas; puede ser el caso, por ejemplo, de las denominadas computadoras personales.

No obstante, existen otras propuestas para la publicación electrónica apoyadas en la idea de que ésta debe soportarse en equipos específicamente diseñados para manejar documentos digitales, tal sería el caso, por ejemplo, de la serie de productos Franklin, 2 o el de la enciclopedia de bolsillo Larousse que es como una pequeña calculadora con un miniteclado que permite buscar 75.000 nombres, 6.000 acontecimientos históricos y hasta 150 listas con dinastías, ríos, obras literarias 0 premios Nobel. Este tipo de tecnologías produce un esquema de publicación con las siguientes características:

* La publicación se realiza a través de un dispositivo electromagnético, generalmente tarjetas de memoria, que sólo pueden utilizarse en un aparato específico, al que se le denomina lector.

* Hastalafecha el diseño de los aparatos para consultar este tipo de documentos digitales tiende a tratar de reproducir las características formales de la consulta de documentos impresos.

* Como no existen estándares en cuanto al diseño de los aparatos lectores, no es factible el uso generalizado de los mismos, cada aparato tiene sus propias características, lo cual implica que una tarjeta con documentos digitales únicamente puede ser utilizada en el lector para el que fue diseñada.

La publicación de documentos digitales que se sustentan en la utilización de equipos de proceso electrónico orientados a la realización de múltiples operaciones, en donde el uso de documentos digitales únicamente es una de ellas,

2 Esta empresa ha desarrollado fundamentalmente productos en el campo de los diccionarios y material didáctico para el aprendizaje de idiomas. Cf. www.com 
como por ejemplo las computadoras personales, podemos dividirla en publicación:

* electrónica como medio para obtener un impreso,

* por medios magnéticos y ópticos, como el disquete o el cd-rom,

* en línea.

Estos tipos de publicación responden a necesidades y finalidades de diversa índole. Sin embargo, en este estudio no nos ocuparemos de la publicación electrónica como medio para elaborar impresos, porque consideramos que ésta representa un fenómeno de reorganización de las actividades de la industria editorial, que si bien cambia los modos de producción de los impresos e influye en su calidad y presentación, el producto final siguen siendo los impresos.

El cd-rom ofrece la posibilidad de organizar y publicar grandes volúmenes de texto, imágenes, sonido e incluso video, aunque no resulta fácil su actualización, puesto que esta depende de una nueva edición. A la fecha se ha utilizado para editar colecciones de textos que se forman con las versiones electrónicas que por medio del escáner se pueden obtener de textos impresos, ${ }^{3}$ pero ha tenido una mayor aceptación en la creación de manuales diseñados específicamente como documentos digitales, en la producción de obras de consulta, y para distribuir todo tipo de software y video juegos.

La publicación en línea utiliza diversos tipos de tecnologías de telecomunicación para cumplir con sus propósitos. Esta forma de publicar crea por medio de las telecomunicaciones, un sistema que le permite al público acceder directamente al documento digital, de manera que se crea un ciclo de edición, publicación, comercialización, en donde el público y el editor pueden establecer variados tipos de comunicación. La publicación en línea puede valerse de tecnologías como los bbsque utilizan una línea telefónica y un modem para conectar una computadora personal al servidor de un editor, o bien aprovechar las redes locales, o las redes de redes como Internet. Como este esquema de publicación depende de la existencia y desarrollo del servicio público de telecomunicaciones factible de utilizarse libremente para estas actividades, su expansión está ligada al crecimiento de este servicio en un territorio determinado.

La publicación en línea encierra una propuesta que permite realizar en un ambiente de automatización y telecomunicaciones todos los procesos relacionados con la publicación, tanto en sus aspectos de diseño, escritura, edición, como en los relativos a su difusión, comercialización y distribución. Representa la opción más

3 La creación deversiones electrónicas de textos impresos recibió un fuerteimpulso con el proyecto Gutenberg, en sus inicios este proyecto pretendía crear un repositorio de textos factible de ser consultado en línea. Sin embargo, lo costoso de un proyecto de esta naturaleza, así como el desarrollo de las tecnologías de la información que favorecieron otros modos de publicación electrónica, hicieron que este proyecto sufriera distintos ajustes, aunque es de destacar el entusiasmo del público para participar en el proyecto, al grado de haber producido gran cantidad de versiones electrónicas de libros clásicos 
acabada de la publicación electrónica, puesto que todos los procesos inherentes a la misma se presentan en un ambiente de telecomunicación.

Cada una de las opciones de tecnología para publicación electrónica encierra sus propias propuestas en cuanto a la naturaleza y forma de los documentos digitales, por tanto determinan el diseño, elaboración y edición de un documento digital. En este sentido, es pertinente hacer notar que el tipo de tecnología a utilizar para construir un documento digital establece la arquitectura y funcionalidad del mismo, así por ejemplo, en el caso de documentos destinados a ser publicados en cd-rom el tamaño de los mismos no es un factor relevante, en cambio, sí lo es para la publicación en línea, puesto que el tiempo de transmisión de un documento es un factor importante, no sólo en cuanto a costos, sino también en cuanto a eficiencia de la transmisión, de ahí que al determinar el tamaño de un documento para su publicación en línea es necesario considerar sus tiempos de transmisión.

\section{Los procesadores de texto}

Uno de los asuntos clave para comprender la idea de la publicación electrónica lo constituye la relativamente rápida aceptación social de los programas para el proceso automatizado de textos, comúnmente denominados procesadores de texto. Existen amplios núcleos de la sociedad para los cuales el proceso de textos por medios automatizados es una parte integral de su trabajo cotidiano, ya sea en actividades de oficina, de índole educativa o de otro tipo. La divulgación del procesador de textos ha sido tan amplio, que prácticamente ha transformado la organización del trabajo en los distintos sectores de la sociedad.

En torno al proceso automatizado de textos, se fueron engarzando algunos fenómenos como el diseño de programas procesadores de texto con una amplia gama de funciones, la disponibilidad de computadoras personales de bajo costo que hacen rentable el proceso electrónico de textos, el incremento de conocimientos orientados a facilitar la aplicación de los procesadores de texto a diversos propósitos relacionados con la investigación, la docencia o la administración, así como el establecimiento y aceptación generalizada de estándares. La presencia de máquinas y programas de bajo costo para automatizar la escritura e impresión de textos, por medio de funciones estructuradas en un solo sistema, conocido como procesador de textos, así como la posibilidad de vincularlos con otros sistemas como manejadores de bases de datos y generadores de documentos html, propició una clara tendencia a crear múltiples documentos y servicios informativos impensables fuera del contexto de recursos que proporciona este tipo de tecnología.

El proceso automatizado de textos se generalizó vía el uso del mismo en la automatización de los trabajos administrativos de diversa índole, los primeros patrones del proceso de texto estuvieron dirigidos a la automatización de actividades de oficina como el manejo de correspondencia, elaboración de manuales de 
procedimientos, entre otros. La automatización de estos procesos favoreció la creación de un esquema de comercialización que abarcó un mercado muy amplio, lo cual propició la rentabilidad de estos primeros productos y su expansión hacia otros sectores, como la docencia, la investigación y la generación de nuevos procesos de producción en la industria editorial.

La creciente utilización del proceso automatizado de textos es un fenómeno que tiende a expandirse en diferentes sectores de la actividad humana. Cada vez el ser humano se va acostumbrando a este nuevo espacio de lectura y escritura, habituándose a introducir sus datos y visualizarlos en una pantalla. La utilización del procesador de textos, con fines de publicación, crece en aquellos aspectos en donde el uso del papel como herramienta de lectura escritura resulta obsoleto.

Es factible observar que a partir de la década de 1970 ha existido un lento y sistemático avance en el uso de la publicación electrónica, en aquellos aspectos en donde el papel impreso no permite un manejo adecuado de algunos documentos; ya sea por la necesidad de actualizar constantemente parte de su contenido, 0 bien, porque el manejo de grandes volúmenes de texto hacen inoperante y costosa la utilización de la imprenta y los circuitos tradicionales de distribución y difusión editorial.

\section{El diseño de documentos digitales}

El diseño de un documento digital, en cuanto a su contenido, no es muy diferente al proceso de creación de cualquier tipo de documento. Sin embargo, la elaboración de su forma, estructura lógica y apariencia, depende de una amplia gama de instrumentos para ayudarse a construir la versión final del mismo. Las herramientas para el diseño de documentos digitales establecen una estructura que en algunas ocasiones favorecen el espíritu creativo, mientras que otras no sólo lo obstaculizan, sino que son una auténtica camisa de fuerza para la creación documental. El diseño y construcción de un documento digital es un proceso que envuelve distintas actividades, cada una de las cuales está dirigida a cumplir un propósito específico previamente configurado por el creador del programa de edición. No obstante, todos los componentes de un documento digital deben estar en concordancia con los procesos de navegación del documento, a fin de que los textos o imágenes guarden sentido al ser visualizados en pantalla por medio de una secuencia de navegación.

$\mathrm{Al}$ igual que en cualquier tipo de documento es necesario establecer un formato para el texto digital, tanto para darle una estructura lógica que facilite la comprensión de los contenidos temáticos que se pretende difundir, como para establecer su apariencia estética. La presentación en pantalla, las fuentes y los encabezados son factores importantes en la organización de un documento digital. Es imprescindible tener en mente que la unidad básica de presentación del documento será la ventana de una pantalla. La idea de la ventana en pantalla como la 
unidad básica de diseño y uso de un documento digital, permite que la persona que opera el programa pueda ver aplicaciones simultáneas que se ejecutan en ventanas independientes unas de otras, este concepto se complementa con el uso del ratón como dispositivo apuntador para tener un control general de la operación de consulta de documentos. Al punto donde se reúnen diferentes ventanas representativas de distintos procesos se le denomina interfaz.

La interfaz de uso de documentos digitales no sólo implica los datos y gráficos que se observan en la pantalla, es decir, la manera en que una computadora muestra los resultados de sus procesos, incluye también los procesos factibles de activarse por medio del ratón, el teclado, o cualquier otro dispositivo, v.g. la sensibilidad de la pantalla. A este tipo de interfaz se le denomina interfaz gráfica G UI (por sus siglas en inglés), aunque para un mortal despreocupado del mundo de la computación pueda no tener mucho sentido, porque en realidad predomina el texto sobre los gráficos. Sin embargo, la palabra gáficodistingue a este tipo de desplegado de pantallas, de las formas de desplegados de pantallas anteriores, las cuales solo presentaban texto, razón por la cual se les denominaba interfaces de modo texto, sería el caso del unix, y el dos

La interfaz gráfica se ha convertido en el medio más aceptado de operar las computadoras personales, v.g. windows95, O S2wrap, y X w de Lynux operan bajo la idea de la interfaz gráfica. Los fabricantes de programas aducen que ésta tiene un manejo más natural y comprensible, lo cual desde luego, resulta trivial, puesto que el aprender a utilizar una computadora, de cualquier clase, requiere de esfuerzo, el asimilar las formas de operación de una máquina implica el mismo trabajo, sea cual sea la interfaz que se use. La ventaja de la interfaz gráfica no tiene nada que ver con los gráficos o con la intuición o naturalidad en la forma de operar una máquina. Ésta es altamente apreciada en el campo de la computación, porque es una tecnología que facilita el colocar en el reducido espacio de una pantalla una amplia gama de funciones fáciles de accionar por medio de dispositivos como el ratón. Lo cual permite incrustar en cualquier lugar de una pantalla una instrucción para accionar una función. De esta manera, para consultar un documento digital lo único que se necesita es apuntar, oprimir el botón del ratón y observar los efectos de esta acción.

\section{ESTRUCTURA DEL TEXTO DIGITAL}

Cualquier documento tiene alguna estructura, inclusive un simple párrafo contiene signos de puntuación que separan oraciones. La estructura de un texto puede deducirse a partir de la distribución del texto en una página impresa, si se conocen los convencionalismos aceptados por una o varias comunidades acerca de la estructura lógica que debe tener un documento. ${ }^{4}$ En términos generales los impre- 
sos se organizan a partir de palabras que forman oraciones, con las cuales se conforman párrafos dentro de una página, que puede dividirse en secciones. Nuestro cerebro asocia la apariencia de un texto con su estructura, muchos de los contenidos de un documento se comunican por medio de su estructura, por ejemplo, el subrayado de una palabra o párrafo puede servir para denotar su importancia 0 llamar la atención, el uso de la palabra "capítulo" nos remite a la idea de una parte específica de un libro. Este marcado o anotación simplifica nuestro entendimiento de un texto y ayuda a evitar ambigüedades. En el caso de los documentos digitales se ha creado lo que se denomina lenguajes de marcado para normalizar la creación de sus estructuras.

\section{Lenguajes de marcado}

El lenguaje de marcado o de anotación comprende un conjunto de reglas que definen todo aquello que es parte de un documento digital, pero que no pertenece al texto del mismo. El lenguaje de marcado cumple con dos objetivos esenciales para el proceso de un documento digital:

* separa los elementos de que se compone un documento digital, y

* especifica las operaciones tipográficas y funciones que debe ejecutar el programa visualizador sobre dichos elementos.

Los elementos son v.g. un párrafo, un capítulo, o cualquier otra construcción que merece ser diferenciada dentro del documento digital. Las operaciones tipográficas son instrucciones de formato aplicadas a cada uno de los elementos, por ejemplo, imprimir un título en itálicas (o cursivas). Existen dos tipos básicos de lenguajes de marcado de documentos: procedural y estructural. Las anotaciones de los lenguajes procedurales describen la forma y el significado de las operaciones tipográficas aplicadas a cada uno de los elementos del documento. Por ejemplo, la regla del lenguaje procedural indicaría que el título de la sección de un texto debe ser impreso en una sola línea con una fuente de seis puntos más grande que el resto del texto, con objeto de que los lectores puedan inferir que es el título. En los lenguajes estructurales las anotaciones únicamente describen la estructura lógica de un documento digital. En general los lenguajes de marcado siguen una sintaxis sustentada en el uso de etiquetas, una etiqueta que indica el principio de un elemento y otra el final del mismo. Las etiquetas se diferencian del texto por medio de caracteres especiales, en el caso del sgml se establece el uso de paréntesis angulares $<>$ para indicar que se trata de una etiqueta de marcado de texto. Un título puede ser anotado como: <Título>El gran libro<Fin_del_Título>, que si bien dice que el título es "El gran libro", no dice cómo debe de aparecer. Las anotaciones estructurales especifican el tipo de elemento y no su apariencia.

Cf. M. D aniel Germán y Alejandro López Ortiz. "SG ML: Lo que está escrito en los espacios en blanco". SduionesA vanzadas, diciembre 1995, n. 28, p. 24-30. 


\section{Investigaaón Biblideedógica v. 12 No. 25 julio/ diciembre de 1998}

El que la estructura de un documento digital se sustente en el marcado por medio de etiquetas puede incrementar las posibilidades de proceso del mismo, debido a que se pueden diseñar funciones sustentadas en el uso de determinados elementos marcados con una etiqueta.

\section{EI lenguaje de marcado SG M L}

El Lenguaje Estándar G eneralizado de Marcado 5 fue establecido por medio de una norma de la O rganización Internacional de Estandarización (ISO), la cual unifica la aplicación de los conceptos de marcado estructural. El SG ML no es un formato de almacenamiento, sino un metalenguaje con el cual se pueden definir lenguajes de marcado con los que se puede anotar el texto digital. El SG ML 6 tiene las siguientes características principales:

* Hace énfasis en las anotaciones estructurales en lugar de las procedurales.

El estándar de SG ML no incluye información sobre cómo deben ser interpretadas las ano taciones. Su principal objetivo es describir las unidades lógicas de un documento, y no cómo debe ser su presentación tipográfica.

* Se sustenta en el concepto de tipos de documentos. El SGML formaliza la idea de tipificar los documentos clasificándolos en clases y especies, al crear la noción de D efinición de Tipos de D ocumentos (DTD). UnaDTD describe una clase o familia de documentos con ciertas características comunes.

* Es independiente del sistema de proceso. El SG ML evita la dependencia de los conjuntos de caracteres, tales como ASCII y EBCDIC, específicos para fabricantes de equipo. El SG ML utiliza caracteres normalmente presentes en casi todos los sistemas y define mecanismos para emplear caracteres especiales o poco comunes.

* Establece un sistema de notación formal para marcar el texto a través de etiquetas enmarcadas con paréntesis en ángulo . Una etiqueta especifica simplemente el comienzo y final de un elemento, un elemento es algo como una sección, párrafo, una frase con texto en tipografía cursiva (itálica), un "ítem" de un listado, etcétera.

* No especifica cómo se debe ver un documento, cada producto que utilice el SG ML define diferentes mecanismos para realizar la transición entre un documento perteneciente a una clase determinada y su versión tipográfica en papel o pantalla.

5 Cf. International Organization for Standarization. Information Processing: Text and office Systems: (SG ML) D ocument Interchange Format (SD IF), 1986. ISO / D IS 9069.

6 Cf. Liora Alshuler. ABC... SGML: A user's gride to structured information Boston: International Thomson Computer Press, 1995. 414 p. Información actualizada sobre el SG ML puede localizarse en los siguientes sitios web:

http:/ / www.w3.org/ org/ hypertext/ WWW/ MarkUp/ SGML y en http:/ / www.sil.org/ sgml/ sgml.htm 
Como los programas para la edición electrónica generan sus propios fomatos es conveniente el uso del Lenguaje Estándar Generalizado para Anotaciones (sgd), como un instrumento para la edición y publicación de documentos digitales. Aun cuando cada tipo de documento requerirá un diferente perfil del lenguaje, sin embargo la aceptación y uso de la sintaxisdd sgnl es un elemento indispensable para lograr un eficaz suministro e intercambio de documentos digitales. El que los autores utilicen el sgml como herramienta para producir sus textos digitales, facilita la edición del texto, puesto que permite convertirlo a distintos formatos, con lo cual no se depende para su edición de un solo producto.

\section{EI lenguaje de marcado H TM L}

El Lenguaje de Marcado de Hipertexto (HiperText MarkupLangaje) fue originalmente diseñado como una Definición de Tipo de Documento (DTD) del SGML. La finalidad del HTML es servir a los propósitos de organizar la estructura de un documento digital en forma de hipertexto, con objeto de que pueda ser fácilmente procesado por un programa visualizador independientemente de la máquina, o el sistema operativo de que se trate. Su amplia difusión se debe a que uno de los estándares que se desarrollaron para Web fue el HTML.

El HTML es un lenguaje constituido por un conjunto de etiquetas para marcar la forma en la cual se deben organizar los contenidos de un documento digital, al ser procesadas por un programa orientado a la interpretación de estas marcas. A estos programas se les denomina visualizadores, existen en el mercado distintas marcas de ellos (Intenert Explorer, Netscape, entre otras) que son los que determinan la apariencia o presentación final del documento en la pantalla de una computadora. El lenguaje de marcado HTML ha seguido evolucionando y se le han introducido nuevas funciones, e incluso subconjuntos que adicionan y aumentan su capacidad de marcado como es el caso del XML. ${ }^{7}$

\section{Lenguaje de marcado $\mathrm{H}$ ytime}

El lenguaje Estructurado de Hipermedia (Hypermedia TimeBased Structuning Langraje, al igual que SGML, se basa en una norma de la ISO, por lo cual ambos lenguajes ofrecen atractivas soluciones para la portabilidad o transferencia de documentos digitales. Se propuso como un lenguaje para representar la estructura de documentos basados en tecnologías como el hipertexto, la hipermedia y la multimedia. Conceptualmente comprende un conjunto de extensiones al SGML, y provee un sistema de marcado estándar para realizar referencias cruzadas. ${ }^{8}$

7 Cf. http:/ / www.arbortext.com/ sgmlresrchtml y http:/ / www.oasis-open.org/ cover/ publicSW.html

8 Peter J. Weingartner, (1997)."A first guide to PostScript." En: http:/ / ibis.nott.ac.uk/ guidelines/ ch3/ chap3-D .html 


\section{4}

\section{Lenguaje de modelado VRM L}

Lenguaje de Modelado de Realidad Virtual (Virtual RealityModdingLangraje) es otro estándar usado actualmente para documentos en Web. Fue creado por Mark Pesce en 1993, con la finalidad de generar, visualizar y ligar imágenes en tres dimensiones. Los visualizadores más populares para el VRML son WebSpace y World View. En principio el VRML se ha utilizado más para cuestiones de entretenimiento, educación y arte. Se considera que en un futuro el VRML proveerá interfaces más transparentes para el usuario, permitirá una mayor interactividad, así como el desarrollo de aplicaciones en colaboración, como podría ser el caso de una conferencia virtual. ${ }^{9}$

\section{Lenguajes de descripción de páginas}

Los lenguajes de descripción de páginas son lenguajes de alto nivel para definir la salida de una impresora. Si una aplicación genera la salida de un lenguaje de descripción de página, ésta puede ser impresa en cualquier impresora que lo soporte.10 Son lenguajes utilizados para la automatización de la producción de impresos, no obstante, nos ocupamos de ellos porque están jugando un papel importante como instrumentos de conversión de datos entre diferentes programas de autor para la publicación electrónica. Es el caso v.g. de adobe acrobat que utiliza el PostScript como medio para importar texto de otro tipo de programas.

\section{Lenguaje T ex}

Es uno de los primeros lenguajes que surgió como un sistema para el procesamiento e impresión de documentos. Fue creado por D onald Knuth de Stanford y puesto al dominio público, tanto el libre uso como la propiedad intelectual. Es un lenguaje que se utiliza frecuentemente para la escritura y publicación de libros, reportes y otros documentos de carácter científico y técnico. Se puede definir como un lenguaje de composición que se utiliza en una gran variedad de sistemas de cómputo y ambientes de composición tipográfica. Utiliza códigos intercalados dentro del texto del documento para dar inicio a los cambios de formato, incluyendo la habilidad de describir fórmulas científicas muy elaboradas.

\section{Lenguaje PostScript}

El PostScript es un lenguaje de programación que se utiliza para lograr un mayor aprovechamiento de las capacidades de las impresoras, sobre todo para la impresión de gráficos. También es conocido como un lenguaje de descripción de

9 Para una mayor información sobre este aspecto consulte las siguientes direcciones

http:/ / www.sdsc.edu/ vrml ó

http:/ / hitl.washington.edu/ proyects/ knowledge_base/ vrlm_bibliography.html

10 Cr. Héctor Jorge Schwabe Mayagoitia. D TP y Postcript. México: el autor, 1995. (Tesis de Maestría: Fundación Arturo Rosenbluet) p. 35 y ss. 
página. Este lenguaje fue introducido por Adobe en 1985, y es de gran utilidad sobre todo para la construcción de documentos con formato pdf.11 Es un lenguaje que genera de manera muy rápida imágenes complejas por medio de instrucciones relativamente simples, permite que formas gráficas arbitrarias sean construidas a partir de gráficos primitivos, tales como líneas y curvas. Además hace muy sencilla la aplicación de fuentes a una página, los caracteres pueden ser escalados (cambio de tamaño), rotados, dispuestos a lo largo de un trazo irregular. D entro de las principales características del PostScript se encuentra el hecho de que es excelente para asegurar la consistencia de imágenes monocromáticas y a color entre diferentes plataformas y medios.

\section{Lenguaje RTF}

El Formato de Texto Enriquecido (RidhText Fomat) fue desarrollado por Microsoft como solución al problema de intercambio de documentos creados en diferentes procesadores de texto y en diferentes sistemas operativos. Éste controla por medio de códigos, las palabras y los símbolos que sirven como un denominador común en los comandos que dan formato a un texto. ${ }^{12}$ El RTF es considerado como un estándar abierto, ya que sus especificaciones se hicieron públicas, y han sido tomadas para otros productos que existen en el mercado de procesadores de texto y de edición como Word Perferct, Interleaf, FrameMaker de Adobe, entre otros.

\section{Filtros y convertidores de formatos}

Los procesadores de texto y los programas para la edición y publicación de documentos digitales procesan los datos a partir de formatos específicos que obedecen a necesidades de programación de cada software en particular, lo cual trajo como consecuencia la presencia de documentos digitales producidos con diversos formatos que sólo pueden funcionar en el ambiente del programa que los genera. Situación que condujo a la incompatibilidad de formatos, a la pérdida de datos en la transferencia, y a dificultades en la conservación y uso de documentos digitales. La solución ha venido de la industria del software que oferta junto con sus productos, programas que permiten el intercambio y manejo del contenido de diferentes formatos de documentos digitales, programas que técnicamente son conocidos con el término de filtrosycanvetidares

11 Cf. Http/ / :grouchy.cs.indiana.edu/ docproyect/ programming/ postscript.html y también: http:/ / www.adobe.com

12 http:/ / www.whatis.com/ rtf.htm 
La idea de contar con filtros y convertidores obedece a eliminar las limitaciones que representan los formatos de cada software. Sin embargo, éstos están estrechamente ligados a determinadas versiones de los programas y también se circunscriben a sistemas operativos específicos, situación que contradice el objetivo de su creación. Por ejemplo, los procesadores de texto se han enfocado al proceso, composición y edición de facsímiles de documentos digitales, pero el uso de un determinado procesador de textos requiere de la utilización de plataformas específicas, y versiones actualizadas. No obstante, los filtros permiten exportar ${ }^{13} \mathrm{e}$ importar ${ }^{14}$ diversos tipos de formatos, que pueden agruparse de la siguiente forma: 15

* Filtros para archivos creados en procesadores de texto y programas de composición. Permiten transferir la información de archivos creados con procesadores de texto y programas para la composición de documentos, que asignan al documento un formato propio del programa en uso.

* Filtros para archivos gráficos. Admiten transferir archivos que almacenan imágenes (dibujos, fotografías, una escena, etcétera) digitalizados en un orden bidimensional, compuesto por elementos llamados pixeles (codificación de bits); o bien objetos creados en otras aplicaciones, v.g. insertar dentro de un documento digital un gráfico, ecuaciones, una hoja de cálculo.

Filtros para archivos de hojas electrónicas o de cálculo. Facilitan transferir archivos provenientes de programas que manejan datos numéricos, texto 0 fórmulas en forma de tablas constituidas por líneas (filas) y columnas, a otra estructura de documento digital.

Filtros para archivos de lenguajes de marcado. Se utilizan para transferir archivos al formato específico de algún software.

Filtros para bases de datos. Su objetivo es facilitar la conversión de datos entre distintos tipos de formatos de bases de datos (dbf, clipper, fox, access, etcétera).

13 Lafunción de exportarpermite que los datos contenidos en un documento realizado en un programa puedan ser guardados con un formato en particular.

14 La función de importar permite que los datos de un formato puedan ser reconocidos porel programa en uso.

15 Véase también la tabla 1. 
La publicación electrónica: ¿Un paradigma de onganización documental digital? 177

\begin{tabular}{|c|c|}
\hline \multicolumn{2}{|c|}{ Tabla 1} \\
\hline Nombre de los formatos & Extensión de los Formatos \\
\hline MSWard RTF & $\mathrm{do} / \mathrm{ntf}$ \\
\hline WardPefet Douments & up \\
\hline FrameMaker InterhangeFomat & mif \\
\hline Ami Professional (ProWritePlus) & sam \\
\hline GldbalV ien XIF & xif \\
\hline Micosatt bitmap & lamp \\
\hline CodDrawDrawing & dr \\
\hline Computer Grapics Meafile & cgm \\
\hline AutoCAD DrawingFileFamat & ding \\
\hline AutoCAD DrawingFomat & $\mathrm{dxf}$ \\
\hline EnhancedWindous Metafile & enf \\
\hline Adbøelllustrator & ai \\
\hline FrameV etor Metafile & fmv \\
\hline CompuServeInterdhangeFomat & gif \\
\hline HP Graphics Langraje & hpg \\
\hline IGES DravingFomat & igs \\
\hline JPEG FileFomat & jpg \\
\hline Kodak Photo & ped \\
\hline AdbbePotableDoament & pdf \\
\hline MacintoshQuidkdraw & pt \\
\hline PostSaip & ps \\
\hline TagImageFileFormat & tif \\
\hline Microsott Windons Metafile & wnf \\
\hline WordPefeet Graphic & upg dp \\
\hline Exc & \\
\hline Laus & \\
\hline Qtropro & \\
\hline
\end{tabular}




\section{Investigaaón Biblideedógica v. 12 No. 25 julio/ diciembre de 1998}

\section{Sistemas de edición}

Al mejorar las funciones y facilidad de operación de los procesadores de texto se fueron derivando sistemas de edición. Un sistema de edición comprende un conjunto de programas que automatizan los procedimientos que ordenadamente se relacionan entre sí para la creación de textos digitales. D entro de un sistema de edición, pueden destacarse los siguientes pasos en el procesamiento de textos: la creación del documento, la edición del texto, el formato del documento, el manejo de los archivos ${ }^{16}$ y la conversión de formatos. Un sistema de edición puede establecerse en una sola computadora para trabajarse por un individuo o un grupo pequeño de individuos, o aprovechar las ventajas de una red, entonces puede hablarse de la existencia de sistemas de edición individuales o en red. Los sistemas de edición se integran por los siguientes elementos: la computadora, programas de procesamiento de textos, gráficos y composición de páginas, y una impresora láser que permitirá imprimir el documento con una apariencia profesional.

Un sistema de edición en red permite aprovechar las ventajas que una red ofrece, como compartir y transferir archivos, encontrar la información requerida, integrarla localmente, manipularla, mejorarla, compartirla y comunicarla. Hoy día es posible entonces concebir la idea de desarrollar un sistema de edición de textos en una Intranet o una Extranet, lo que deviene en el concepto de publicación en línea.

\section{E dición de documentos digitales}

Para darle forma a un documento digital, con objeto de facilitar su difusión transferencia y disfrute, es factible utilizar distintos tipos de tecnologías, cada una de las cuales ofrece diferentes soluciones al problema relacionado con las formas para estructurarlo, generarlo y difundirlo. En términos generales las tecnologías que permiten estructurar y generar documentos digitales con la finalidad de facilitar su distribución, determinan la naturaleza y forma de uso del documento digital, ${ }_{17}$ y establecen los procesos y requerimientos de edición del texto digital utilizado para configurar la versión final de un documento digital.

El significado de editar está correlacionado con la idea de publicar, en tanto que la acción esencial de la edición de un texto estriba en estructurarlo para darle forma de acuerdo con los criterios establecidos para caracterizar determinado tipo de documentos. Tradicionalmente la edición ha sido una actividad independiente y posterior a la escritura y corrección del texto, no obstante, con la intro-

16 Horacio Martínez, pp. 35. Autoedián Santafé de Bogota; Colombia: CERLAC, 1992. 66 p.

17 En el mercado existen diversos tipos de productos para que un autor 0 un editor puedan configurar la versión final para la publicación de un documento digital, entre otros of AddbeAadbat, www.adobe.com/ acrobat; Nebbok www.neosoftware.com/ neosft/ 
ducción de los procesadores de textos es factible realizar estas dos actividades tanto en forma simultánea como independiente una de otra.

La tecnología para el proceso automatizado de texto hace flexible la organización de las actividades necesarias para escribir, estructurar y preparar la publicación de un documento. La flexibilidad en cuanto a la organización de las actividades para escribir y editar un texto, sólo es comprensible en relación con la estructura secuencial y rígida de producción de textos que impuso la imprenta, al mecanizar el proceso de reproducción de un manuscrito. Los procesadores de texto retoman las formas de producción de textos impresos y al automatizarlas establecen la posibilidad de una organización flexible de las formas para producir un texto digital.

La flexibilidad brindada por los procesadores de texto para organizar las actividades relacionadas con el diseño y escritura de un documento digital, hace que la edición electrónica adquiera un sentido distinto de la forma tradicional impuesta por la imprenta. En primer término es necesario destacar que la ductilidad para organizar la producción de facsímiles de un documento digital deja abierta la posibilidad de crear varios facsímiles dependiendo del tipo de componentes que intervengan en su composición. En términos generales un procesador de texto como WordPerfect, Word, Notabene, entre otros, permite automatizar en diversos grados algunos aspectos de la escritura de documentos, para hacer factible:

- construir patrones de estilo, o utilizar los construidos para estos propósitos, con objeto de dar forma al documento que se escribe, de tal manera que al momento de la escritura el programa organice el texto conforme a la estructura deseada,

* automatizar algunos aspectos de la corrección ortográfica y gramatical del texto, la corrección puede realizarse en forma simultánea a la escritura 0 posteriormente a la misma,

* combinar la escriturade textos, con el aprovechamiento detextos almacenados en distintos formatos, desde sus formas más simples, como puede ser la producción de cartas personalizadas a partir de algunos patrones de carta y una base de datos de direcciones, hasta formas más complejas como el utilizar una base de datos de fichas de textos sobre tópicos específicos para producir una secuencia de textos ordenados temáticamente,

* operar en forma sincrónica con el procesador de textos, algún otro tipo de programas para añadir al texto diagramas, imágenes, sonido, color, video,

* producir distintas versiones de un documento, para su difusión en papel, en línea o por medio de dispositivos electromagnéticos,

* automatizar la construcción de índices, tablas de contenido, citas bibliográficas, notas, referencias bibliográficas, ligas al web. 
Al organizar la producción de un documento digital, se puede optar por usar 0 no cada una de las funciones descritas anteriormente, o formar una combinación de ellas, asimismo se puede controlar la traza tipográfica del documento, o bien dejar que los programas que visualizan o imprimen documentos determinen su apariencia. Al seleccionar las funciones que habrán de utilizarse para producir el documento digital, se configura la naturaleza del mismo, y se da lugar a generar distintos tipos de facsímiles para un mismo documento digital.

Un autor puede crear un facsímil que contenga anotaciones conforme a la definición del lenguaje de marcado para documentos digitales conocido como sghl, con lo cual el facsímil del documento contiene los elementos necesarios para reproducir tanto la estructura lógica como el aspecto tipográfico del texto digital. La decisión acerca de controlar o no la apariencia del documento digital, establece el modo de la edicónypublicadón detrónica, puesto que el difundir un documento digital conservando en todo momento su estructura, implica un modo de edición que requiere el uso de tecnologías específicas para tal fin.

No sólo la decisión acerca de la posibilidad de controlar o no la apariencia final de un documento digital establece el modo de edición y publicación del mismo, la tecnología que se adopte para confeccionar la estructura del documento digital es un factor que fija incluso la forma de escritura del mismo. Es, por ejemplo, el caso de la tecnología del hipertexto, el sustentar el diseño y construcción de un documento en este tipo de tecnología hace necesario que las partes lógicas de que se compone el documento, al momento de redactarse, tengan algún significado por sí mismas, para que el significado de una parte del texto pueda enlazarse al significado del texto de otra parte, a fin de aprovechar las bondades de la tecnología. Lo cual obliga a escribir el documento de una manera acorde con la tecnología, dado que los textos habrán de tener un significado por sí mismos y contribuir a lograr un nuevo significado al relacionarse con otro. Incluso el uso del hipertexto permite la posibilidad de editar documentos digitales escritos por diversos autores y establecer enlaces y relaciones entre ellos, de forma que varios documentos independientes entre sí pueden ser unificados para constituir otro documento. 18

La tecnología para componer un documento digital puede ser heterogénea, e imprimirle variadas características en cuanto a su apariencia y funcionalidad. En términos generales los productos que se encuentran disponibles para publicación electrónica combinan diversos tipos de tecnologías para construir un documento digital, y tienen en común el que requieren la realización de procesos de edición onientados a adicionarle al texto digital aquellas características de marcado y formato ne-

18 Cf. Mercedes Caridad y Purificación Moscoso. Lossistemasdehipetextoehipemeelios UnanuevaAplicadón dela informática doumental- Madrid: Fundación G ermán Sánchez Ruipérez, 1991.- 153 p.; y G eorge P. Landow, - Hipertexto: Laconvegeniadelateena úíticacontemparáneaylatendoǵa- Barcelona: Paidós Ibérica, 1995.- 284 p. 
cesarias para su proceso por parte de los programas que han de servir para visualizar el documento en una pantalla o imprimirlo en papel.

En términos generales la automatización del texto obliga al uso de reglas de escritura orientadas a lograr un mejor aprovechamiento de la tecnología utilizada. Como en el entorno de la publicación electrónica, el visualizar un documento se desplaza hacia la pantalla de un monitor, el diseño de las pantallas se convierte en un elemento fundamental, tan básico como el diseño de una página en un impreso, ya que de ello dependen no sólo las facilidades para consulta y uso de un documento digital, sino también el que las personas que habrán de utilizarlo puedan hacerse una idea de conjunto acerca de la naturaleza, contenido y alcance del mismo.

Los programas para publicación electrónica establecen patrones para configurar la visualización de un documento digital. No obstante, al diseñar un documento digital, es indispensable considerar los siguientes asuntos:

* La única manera de saber acerca del contenido, tamaño y alcance de un documento digital es a través de lo que se muestra en una pantalla.

* La navegación de un documento digital para leer, consultar o extraer información del mismo depende de las funciones que se agreguen para tal fin. G eneralmente estas funciones dependen de dispositivos como el teclado 0 el ratón, lo cual implica tener presente que la operación de un documento digital está sujeta al grado de habilidades para manejar estos dispositivos. Siempre hay que tener presente que demasiadas opciones de funcionamiento pueden contribuir a confundir más que a facilitar la navegación del documento.

* Para recorrer el texto en la pantalla de un monitor es factible hacerlo línea por línea (scroll) o presentar bloques de texto por medio de la función de avance y regreso de página. Ambas formas producen resultados distintos cuando se hace la consulta. Inclusive es necesario tenerlas en consideración al momento de establecer el tamaño de los párrafos de un texto.

* El diseño de la forma de acceso al contenido del documento debe estar orientado a facilitar tanto la consulta como a obtener una idea acerca de la estructura, tamaño, forma y contenido del documento, teniendo en consideración que la primera pantalla es el único medio que tiene una persona para hacerse una idea del documento. D ependiendo de la tecnología que se utilice existen diversas opciones para organizar el contenido de una pantalla. Yasea en forma de menús para ordenar el acceso al contenido del documento en varios planos, ya sea dividiendo la pantalla en sectores, utilizando menús flotantes.

- La tipografía debe atender a las características de los monitores. No siempre la tipografía que da buenos resultados en los impresos produce una visión legible y agradable en una pantalla de monitor. 


\section{Investigaaón Biblideedógica v. 12 No. 25 julio/ diciembre de 1998}

Los programas de edición procesan el facsímil del documento digital para convertirlo en una estructura con las marcas suficientes para poder visualizarlo en la pantalla de un monitor, y/ o producir un original tipográfico con todo o partes del contenido del documento. El documento digital que se origina sólo es factible manejarlo por medio de programas que reconozcan esa estructura, razón por la cual la edición de un documento para conferirle una estructura específica condiciona el empleo de programas que la admitan.

La edición de un documento digital puede realizarse por el propio autor valiéndose de las denominadas herramientas de autor para publicación electrónica 0 bien entregar a un editor un facsímil del documento digital. En términos generales, los editores tienden a solicitar y reciben originales escritos en procesadores de texto, con el formato necesario para que el editor pueda utilizarlos para la producción de un documento digital.

Como resultado del proceso de edición se obtiene un facsímil anotado con las marcas suficientes para producir la versión final para publicación de un documento digital. Como este facsímil anotado puede corregirse fácilmente, e incluso agregarle características nuevas al documento, nos encontramos que mientras el proceso de edición para la imprenta tiende a fijar de una vez y para siempre las características de un impreso, en la edición de un documento digital para publicación electrónica se produce un facsímil flexible del documento digital, que puede utilizarse para variar la estructura y apariencia del documento digital sin necesidad de modificar el texto que contiene. Lo cual da lugar a que un documento digital pueda actualizarse fácilmente, dada la flexibilidad del facsímil que sirve para obtener copias del mismo.

Un análisis del proceso de edición de un documento digital puede dejar claro que es un proceso que puede distorsionar y cambiar los significados, intenciones y mensajes a transmitir, puesto que produce un documento que adquiere las características de la tecnología que sirve para producirlo. Lo que invita a la reflexión en el sentido de aquilatar la importancia de la edición de un documento digital, ya que la aplicación de procesos sin sentido, únicamente para aprovechar las características de la tecnología que se utiliza, puede conducir a documentos digitales confusos, difíciles de usar y con poco valor informativo.

Con la edición cuidadosa de un texto digital, el usuario final gana opciones, pero sobre todo información confiable, en cuanto al sentido que su autor(es) desea(n) transmitir. Podrá obtener documentos impresos en papel, pero tendrá asimismo la posibilidad de disponer de la versión electrónica de los documentos anotados con un lenguaje de marcado (sgml) que facilite su proceso automatizado con los fines que se considere convenientes, pero teniendo a la mano la idea original del autor al momento de generar el documento. 


\section{Programas de autor}

Los denominados programas de autor para publicación electrónica son sistemas cerrados dirigidos a simplificar la edición, construcción e integración de un documento digital, a fin de compilarlo como una aplicación completa en una sola unidad, es decir, integran en una sola estructura y en un solo formato, los diferentes elementos de que se compone un documento digital, como puede ser texto, imágenes, sonido y video. ${ }^{19}$ Asimismo permiten el diseño de la presentación que habrá de tener cada una de las pantallas del documento, y agregarle los instrumentos de navegación, formas de cambio de pantallas, ligas, rutinas de búsqueda, etcétera. En términos generales contienen funciones que permiten incluir en el documento:

* Formatos para determinar la apariencia de las pantallas del documento.

* Formas para buscar dentro de los contenidos del documento.

* Sistemas de navegación y ligas entre los componentes del documento para facilitar su revisión y consulta.

- Procesos para administrar colecciones de documentos.

* Filtros y convertidores para transferir los contenidos de los documentos.

* O pciones de seguridad de los contenidos del documento, que facilitan la introducción de claves para impedir la modificación y uso del documento si no se cuenta con la clave respectiva.

Los programas de autor son sistemas útiles para el diseño y construcción de la arquitectura del documento, es decir, la forma y apariencia que debe tener. Parten de la idea de que los contenidos del documento, es decir, textos, imágenes u hijas detrónicas o de cálaulo ${ }^{20}$ fueron construidos por algún otro medio. G eneralmente los programas de autor no cuentan con procesadores de texto, programas de cálculo o graficación. Aunque es factible que en algunos programas de autor se incluyan funciones de corrección del texto éstas tienen que realizarse a través del enlace con las funciones de un procesador de texto. Es importante señalar que sus funciones están dirigidas a la edición del documento, es decir, a darle forma para su publicación, lo que presupone que el texto ya está previamente escrito para poder editarlo.

El tomar cualquier texto, imagen, video u hoja electrónica para conformar un documento digital mediante el uso de programas de autor, hace patente el problema de la migración de los datos de un formato a otro. Dado que es factible la pérdida de información y fidelidad en el caso de las imágenes, algunos programas de autor contemplan diversos componentes, que proponen algún tipo de solución al

19 cf. http:/ / www.w3.org/ amaya; para documentos acrobat véase: www.adobe.com y http:/ / emerge.pdfzone.com/ cf. También http:/ / www.arbortext.com/ sgmlresrc.html

20 Conocidas también con el término de hojas de cálculo, se refiere alos programas que manipulan tablas constituidas por líneas o filas y columnas que contienen datos numéricos, de texto o manejan fórmulas, cuya característica principal es la presentación de esta información en una pantalla. 
problema de transferencia de documentos, como es el uso de convertidores o filtros.

Un documento digital requiere que se diseñe la forma de presentación de cada uno de sus elementos, con objeto de que las pantallas tengan una apariencia que facilite la consulta y cumpla con los objetivos de comunicación del documento, es decir, que no sólo tenga una bella apariencia sino que ésta contribuya a decir "algo" al sujeto que la ve. Una de las finalidades de los programas de autor es facilitar el diseño y construcción de estas pantallas como un todo integrado, a fin de darle al documento unidad.

La comprensión de un documento digital por parte del lector requiere de funciones que posibiliten el apreciar en su conjunto las partes de que se compone, en otros términos podríamos decir que necesita de funciones que cumplan los mismos objetivos que la tabla de contenido y los índices en un impreso. Como la tecnología que sustenta el diseño, construcción y manejo de un documento digital tolera distintos tipos de búsqueda, es conveniente aprovecharla y diseñar procesos para averiguar a través de tablas de contenido, índices, notas, figuras, el contenido del documento en cuestión; así como dentro de las palabras y frases de que se compone el texto incluido en el documento. Los medios para revisar y acceder a cada una de las partes de un documento digital constituyen el sistema de navegación del mismo, y su objetivo es guiar al público en la consulta del documento.

Los programas de autor contienen una serie de herramientas para construir e integrar las funciones necesarias para navegar en un documento, funciones que pueden adoptar la forma de sistemas de búsqueda, índices, enlaces de hipertexto, etcétera.

\section{V isualizadores}

Los visualizadores también conocidos como lectores o navegadores son programas cuyo objetivo es permitir el uso de documentos digitales estructurados conforme a un formato específico. Contienen funciones para visualizar en pantalla los diferentes componentes de un documento, de acuerdo con las instrucciones agregadas al documento en el momento de la edición. D ependiendo del tipo de producto pueden ser programas independientes del documento digital, como el caso de Acrobat, o bien, encontrarse integrados en una sola unidad con el documento.

\section{LA COMERCIALIZACIÓN DEL DOCUMENTO DIGITAL}

La naturaleza del documento digital ha creado nuevas formas de comercialización y organización documental, porque su estructura, sustento físico y modos de circulación y difusión son diferentes de los establecidos en torno a la imprenta, incluso podríamos decir que representa un fenómeno inédito que modifica las formas tradicionales de compra venta de documentos, al igual que los derechos 
de autor y la propiedad intelectual. Entre otras cosas, altera los servicios bibliotecarios, por ejemplo, no puede aplicarse al documento digital la idea de préstamo bibliotecario, dado que prestar un documento digital significa transferirlo al lector, quien puede quedarse con él, por lo cual los editores de publicaciones electrónicas aducen que este tipo de servicio anula la actividad editorial. En este sentido las bibliotecas han optado únicamente por referir al público al sitio de publicación, con lo cual desaparece la esencia básica de la biblioteca como formadora de colecciones documentales.

En el caso de la publicación en línea el público tiene acceso directo al repositorio documental del editor, desde cualquier parte donde cuente con una línea telefónica, un modem o una comunicación de red, podríamos decir que las 24 horas del día, y si cuenta con los recursos económicos suficientes, puede transferir de inmediato el documento a la máquina donde se encuentre, aunque en algunos casos se dispone de información que puede transferirse libremente. Situación que si bien simplifica la adquisición documental, genera por otro lado la necesidad de medios catalográficos para enterarse de lo que se encuentra disponible.

Aun cuando asistimos a un fenómeno sumamente avanzado y con una gran presencia en determinados círculos sociales, aún dista mucho de constituir un paradigma totalmente acabado, quedan muchos problemas por resolver en distintos aspectos, tanto de la producción y organización documental, como en el manejo de los derechos de autor y la propiedad intelectual. Sin embargo, de continuarse por el camino que se transita es indudable que representa una nueva propuesta en la comercialización y organización documental, que afectará la forma en que se generan y aprovechan actualmente todo tipo de documentos.

\section{Publicación en medios magnéticos y ópticos}

Aun cuando una parte considerable de la diseminación de documentos digitales se realiza por medio de dispositivos electromagnéticos como cintas magnéticas, disquetes, discos zip, ha sido el cd-rom el que ha servido para desarrollar un modo de publicación electrónica, en donde el manejo de grandes volúmenes de información constituye el eje básico del interés de publicación, como es el caso de bases de datos, manuales sobre múltiples temáticas, obras de consulta, video juegos y películas. Asimismo se ha prestado para la publicación de un nuevo tipo de documentos denominados miltimedia, cuya arquitectura considera el uso de texto, gráficos, sonido, imágenes e incluso video. Estos documentos generan archivos de datos muy grandes y por tanto requieren del uso de dispositivos de alta capacidad de almacenamiento a bajo costo, como es el caso del cd-rom; en este sentido los productores de obras de consulta como enciclopedias y diccionarios especializados, lo han aprovechado para incursionar en el terreno de nuevas estructuras para este tipo de documentos, sobre todo a partir del modelo creado por Encarta 
de Microsoft. También se ha utilizado con éxito en el diseño y producción de obras de carácter didáctico.

Aun cuando el cd-rom en sus inicios se utilizó como un instrumento para editar colecciones de impresos, así como de archivos con información de todo tipo, con el tiempo ha ido adquiriendo un espacio propio en el mundo de la publicación, como es el caso del video juego, de las bases de datos, de los multimedia, de la compilación de artículos de revistas, o incluso como dispositivos de almacenamiento anexos a determinado tipo de libros y revistas. La publicación en forma de cd-rom, una vez pasado el impacto que significó la posibilidad de agregar grandes volúmenes de información en un solo dispositivo, ha tenido que evolucionar y enfrentar los problemas relativos a la organización, recuperación y disponibilidad de la información contenida en uno o varios cd-rom.

Hasta ahora los productores de cd-rom han apoyado el diseño de sus productos en las bondades derivadas de la enorme capacidad de almacenamiento de estos dispositivos y por lo general el diseño de su interfaz de uso, procedimientos de instalación y organización de la información dejan mucho que desear. Pocas publicaciones en cd-rom están diseñadas para utilizarse en su totalidad en el cdrom, la mayor parte requiere de cargar programas en el disco duro de la máquina en donde se pretende hacer uso de ellas, lo cual en muchos casos las hace poco eficientes. La mayoría de estas publicaciones ha encontrado mercado al amparo de instituciones como las bibliotecas y grandes empresas, fundamentalmente porque han solucionado problemas relativos a la disponibilidad de información, como en el caso de las bases de datos bibliográficas, o a problemas de almacenamiento, como en el caso de las colecciones de artículos de revistas. Sin embargo, las colecciones de cd-rom son de difícil manejo, por los costos de infraestructura que suponen, y porque crean sobreabundancia de información, muchas veces redundante y con escasos instrumentos para su acceso.

Algunos productores de cd-rom apelan en su publicidad a la idea de que éste constituye una biblioteca electrónica, aunque en términos estrictos sea una afirmación falaz, podríamos decir que expresa el significado del cd-rom como instrumento de publicación, en tanto que se hace alusión a su capacidad de almacenaje, pero también a la necesidad de crear nuevas formas para organizar el acceso a la información que contiene. No obstante está adquiriendo un importante papel como medio de organización y preservación de colecciones de documentos digitales, sobre todo a partir de la aparición de grabadoras de cd-rom que pueden funcionar como periféricos de una PC y es factible escribir varias veces en un solo cd-rom. No obstante, el cd-rom ha creado nuevos problemas para su manejo, ya que sus contenidos en algunas ocasiones incluyen diversidad de contenidos temáticos, puesto que reúnen diversos tipos de documentos en un cd-rom, los que resulta complejo rescatar cuando se conforman colecciones de los mismos. 


\section{Publicación en línea}

El desarrollo de las telecomunicaciones y el uso de protocolos estándar para la transferencia de archivos entre dos o más máquinas, facilitó el establecer sitios de acopio y difusión de documentos, con lo cual se abrió la posibilidad de la publicación electrónica en línea, y de nuevas formas de acopio y uso de documentos digitales, al motivar el que distintos individuos depositen documentos en una máquina, mismos que pueden ser transferidos a otra máquina por otras personas, para darles el uso que consideren conveniente.

El crecimiento de instalaciones de redes de telecomunicación, tanto de uso público como privado, está transformando rápidamente los hábitos de comunicación de algunos sectores de la sociedad. El fax, el correo electrónico, las redes de telecomunicación locales, regionales y globales como Internet, inducen nuevas formas en cuanto al uso y transmisión de texto digital, a la vez que dan lugar a una amplia gama de actividades comerciales en cuanto a la prestación de todo tipo de servicios de información.

Las redes de telecomunicación y el correo electrónico han generado condiciones sociales que están creando nuevos espacios para la comunicación, no sólo de información sino incluso de archivos completos con diversidad de contenidos, con lo cual se modifican los hábitos acerca de las formas de acopio de información, y maneras para generar conocimientos, con lo cual paulatinamente se establecen nuevos procesos sociales para organizar y transmitir información y conocimientos. A este respecto, podemos mencionar:

* los repositorios de archivos digitales con contenidos temáticos sobre tópicos específicos, denominados ftpen alusión al protocolo de transmisión de archivos que los hace posible,

* las páginas web,

* las listas de correo electrónico y la compilación de los mensajes de éstas,

* los archivos depreguntasy respuestas más frecuentes sobre un tema(faq),

- los foros de las redes.

Estos nuevos modos de comunicación de información y conocimientos representan un producto típico del uso de las telecomunicaciones y la automatización como medio para generar otras formas para la circulación de contenidos temáticos de todo tipo, organizados en forma de archivos o documentos digitales que aprovechan la automatización para facilitar el uso y sistematización de información. Hasta ahora, el desarrollo de estos productos ha seguido los esquemas y criterios que tanto la industria editorial como las organizaciones académicas y de investigación mantienen para validar las publicaciones impresas, incluso una de las primeras ideas de la publicación electrónica estuvo orientada a digitalizar los impresos. No obstante, ha prevalecido la difusión de documentos digitales y formas de publicación electrónica, sustentadas en la idea de los servicios informativos, es decir, el sujetar la publicación en línea al concepto de transferencia de datos ac- 
tualizados, que ha tenido un mayor éxito y desarrollo sobre todo a partir de la difusión del www.

La presencia de estos fenómenos, en cuanto a las posibilidades de difusión de información, está creando nuevos entornos en cuanto a los criterios y convencionalismos sociales respecto a las formas de validar los documentos que circulan en estos medios. Sin embargo, es necesario fortalecer los métodos y conceptos de la tipología de documentos digitales, con objeto de contribuir con elementos que ayuden a caracterizar a esta clase de documentos, y con ello coadyuvar a lograr una mejor calidad de los mismos. Al inducir el análisis constante de las características y finalidades de los documentos digitales se podrán generar valores que sirvan como criterios para su evaluación.

No hay que perder de vista que la publicación electrónica introduce la idea de la actualización parcial de un documento digital, sin necesidad de modificar toda la estructura del mismo. Aunada a esta idea, se encuentra el fenómeno de la disponibilidad para su consulta, casi al mismo tiempo en que ésta se realiza, es por ejemplo, una de las finalidades que se persiguen con la publicación electrónica por medio de los www. De tal manera, que nos encontramos frente a un producto editorial que tiene una serie de características que lo conforman como un producto acabado, pero que a diferencia de un impreso, éste se puede actualizar fácilmente, eliminando la información considerada obsoleta. En otros términos, la publicación en línea pretende mantener una publicación siempre actualizada, sin necesidad de modificar la estructura y finalidades del documento, sólo añadiendo lo que se considera nuevos contenidos, sin necesidad de conservar registro de lo que se suprime.

Si la capacidad de actualización derivada de la publicación en línea se lleva a sus últimas consecuencias entonces nos encontraremos conque el sitio de publicación y sus formas de acceso y disponibilidad serán elementos fundamentales para evaluar la calidad de la publicación. El que exista la posibilidad de la actualización parcial no quiere decir que la publicación en línea tenga necesariamente que generar productos de esta naturaleza.

La idea de manejar información novedosa y desechar aquella que resulta obsoleta se introdujo junto con la idea del desarrollo científico y tecnológico como instrumentos para lograr una eficiente forma de control y manejo de la publicación de información científica y es uno de los elementos esenciales de la publicación electrónica para proveer a los usuarios de alternativas diferentes en cuanto a formatos, costos y calidad de la información, para obtener documentos electrónicos. 


\section{LA CONSTRUCCIÓN DE UN ORDEN DOCUMENTAL DIGITAL}

Asistimos a un nuevo fenómeno en cuanto al aprovechamiento de las tecnologías de la información para registrar y difundir información, por medio de un nuevo tipo de documento con sus propias características, distintas de las de la imprenta. Actualmente un documento producido originalmente por medios digitales puede sufrir constantes transformaciones sustentadas en las facilidades derivadas del proceso automatizado de textos, puesto que es factible utilizar sus textos como parte de otros textos, o bien anotarlos y producir distintas versiones de un documento, pudiéndose presentar el caso de que cambie el significado de los mensajes del documento, al transferirlo de un formato a otro. Mientras en épocas anteriores el único proceso de aprovechamiento del documento se producía por medio de la lectura y comprensión del mismo por parte de un sujeto, ahora se tiende a generar patrones de proceso de información que realizan las máquinas y sus programas en forma automática, v.g. el caso de la construcción automática de índices por parte de los buscadores en el web.

Como la tecnología que da lugar a la producción de documentos digitales aún no alcanza el grado de madurez deseado, no es fácil de utilizar para el público neófito en estas cuestiones, se ha dado lugar a una corriente de tecnorromanticismo que le adjudica toda clase de bondades. Sin embargo, el mayor desafío de esta forma de producción y organización documental se encuentra en el diseño de productos de software, que faciliten y simplifiquen el diseño y creación de documentos digitales por parte del público para fines docentes y de investigación, dado que hasta ahora se ha dirigido en mayor medida a facilitar el manejo de la documentación administrativa de las grandes corporaciones, produciendo sistemas y programas cuyo costo resulta elevado para su aplicación individual.

La transición social del documento impreso al digital requiere no sólo de una amplia comprensión del fenómeno, sino de una participación activa por parte del bibliotecólogo y de los sistemas de docencia e investigación, en el sentido de replantear los parámetros de la tecnología que hemos descrito en párrafos anteriores, abundando en la idea de que el documento digital no sólo requiere de soluciones tecnológicas que faciliten su programación, sino además de resolver los problemas referentes a las relaciones de significación y valoración que se dan entre un sujeto y un documento. La significación de los contenidos documentales está sustentada en el valor que le atribuye el público, por tanto es indispensable introducir en la discusión de las estructuras del documento digital, las relativas a las formas de organización y preservación de los contenidos temáticos para salvaguardar no sólo la continuidad de las formas para construir el conocimiento, sino de la libertad para poder hacerlo.

El papel de la publicación electrónica no puede ni debe restringirse a ser un mero mecanismo de reproducción o de difusión de los impresos ya publicados, porque con la digitalización de impresos se contribuye a distorsionar la organiza- 
ción documental al crear acervos, que si bien solucionan en parte el acceso a los documentos, no contribuyen en nada a establecer un nuevo orden documental, puesto que únicamente se trasladan documentos de un medio a otro, y además encarecen los costos para el público usuario, al tener que imprimirlos para poder consultarlos, pues el documento resulta de difícil lectura en una pantalla. Además, si junto con la digitalización de impresos se introducen técnicas de indización que recuperen fragmentos de impresos digitalizados, se está contribuyendo a crear confusiones de significados y falsos conocimientos, pues los programas de recuperación entregan al usuario fragmentos de información fuera de contexto, perteneciente a documentos que fueron concebidos por sus autores como un todo unitario, ya sea en forma de libro o de artículo de revista.

A nuestro modo de ver, la publicación electrónica representa alternativas para crear un orden documental que ayude en el ámbito de la investigación y la docencia a generar procesos que contribuyan a la enseñanza aprendizaje del manejo documental, dado que puede servir para inducir al estudiante al análisis de los métodos de diseño y difusión documental. ${ }^{21}$ Asimismo puede ser provechosa como medio para promover la cooperación en la investigación, mediante la creación de redes académicas que fijen criterios actuales, en cuanto a la forma de arbitrar y evaluar la información que como resultado de la investigación sea factible difundir por medio de estas redes. Lo cual implica abordar el problema del significado en la organización de los contenidos temáticos de los documentos, de la organización de sus mensajes y su preservación, desde el punto de vista del aprovechamiento de la información por parte del sujeto que en un momento dado puede requerirla. D e otra manera estaremos aceptando sin cuestionar la construcción de programas que indudablemente tienden a generar un nuevo orden documental sustentado en la robotización en el manejo de la información, por medio del uso de formularios ${ }^{22}$ para ordenar y jerarquizar el contenido y los mensajes a introducir en un documento digital, con lo cual se verá profundamente empobrecida nuestra capacidad no sólo para formar acervos documentales benéficos a los propósitos de mejorar las condiciones de vida de una comunidad, sino incluso estaríamos hablando del empobrecimiento del conocimiento humano.

Hasta ahora la discusión acerca de la publicación electrónica, los documentos digitales y la posibilidad de un nuevo orden para la organización documental se ha presentado en torno de las estructuras de programación necesarias para configurar sistemas para el diseño y transmisión del documento digital, dejan-

21 Cf. Carlos Serrano Cinca. "El world wide web en la docencia universitaria." - En: Tendenaasdelain vestigacónendbamentacoón- Editado por Jesús Tramullas. Zaragoza, España: Seminario TID , 1996.147-160.

22 Los formularios de estilo son de uso común en los procesadores de texto. Sin embargo, están sustentados en los conocimientos acerca de la estructura lógica de los textos. En cambio en los formularios para la publicación digital prevalece la idea de trasladar estructuras de documentos de tipo administrativo para aplicarlas en el ámbito de la docencia e investigación. 
do de lado que un documento digital no es un formato o una estructura, sino un conjunto de mensajes con un destinatario. Poco se alude a la influencia de la estructura tecnológica en los significados, contenidos temáticos y mensajes de este tipo de documentos y por tanto en el orden y jerarquización que debería adoptarse para la configuración de sus mensajes, en algunos casos, se piensa que únicamente cambia el medio y no se altera la naturaleza del mensaje. Empero, si analizamos cuidadosamente todas las estructuras y fórmulas tecnológicas implicadas en una posible organización documental de corte digital, es relativamente fácil percatarse de que no sólo se afecta la forma, apariencia y funcionamiento del documento digital, sino también la manera, contenido y proceder de interpretación de los contenidos.

La publicación electrónica conlleva una oferta tecnológica que hace necesario estudiar a fondo la naturaleza de los mensajes construidos a través del documento digital, con objeto de evitar reducir la solución de los problemas de la publicación electrónica a cuestiones meramente tecnológicas, ajenas a la representación de los contenidos temáticos de los mensajes que se quiere comunicar. Sería fructífero profundizar en el estudio de las estructuras del documento digital en relación con el tipo de mensajes que se pueden manejar, pero sobre todo en la manera en que los sujetos los valoran e interpretan, como un primer acercamiento, para desprender principios que nos permitan realizar sugerencias acerca de cómo valorarlos y ordenarlos en forma de acervos de servicio público que contribuyan a fortalecer la docencia e investigación y la difusión de información y conocimiento en favor del mejoramiento de las condiciones de vida de la sociedad en general.

\section{REFERENCIAS ELECTRÓNICAS}

Abordar el tema de la publicación electrónica no sólo requiere acudir a fuentes de consulta impresas, sino que es necesario también referirse a las fuentes de información en línea. Son diversos los sitios web que existen sobre estos asuntos, de ellos se han seleccionado aquellos cuya característica principal es su actualización.

El anexo que se presenta es el resultado de la búsqueda y organización del material que sobre publicación electrónica consideramos de mayor relevancia. A través de éste será posible encontrar sitios de interés que conducen a documentos electrónicos y que en algunos casos forman parte de lo que comúnmente se le ha denominado colecciones digitales o bibliotecas virtuales. Las referencias están organizadas de acuerdo con los temas tratados en este artículo, pensamos que en determinado momento es más conveniente tener el URL del sitio como una fuente de información más que el nombre y dirección de un documento específico. 


\section{Lenguajes de marcado}

http:/ / www.adobe.com (Adobe Systems Incorporated)

http:/ / www.csdl.tamu.edu/ ohs/ (O pen Hypermedia Systems Working G roup)

http:/ / www.w3.org/ (The World Wide Web Consortium)

http:/ / www.ncsa.uiuc.edu/ SD G / Software/ Mosaic/ WebSG ML.html (SG ML on the Web)

http:/ / www.ncsa.uiuc.edu/ SD G / Software/ Mosaic/ D ocs/ d2-htmlinfo.html (Creating HTML D ocuments)

http:/ / www.sgmlopen.org/ (OASIS : O rganization for the Advancement of Structured Information Standards)

http:/ / www.arbortext.com/ xmlresrc.html (XML Resources - ArborText: Software for SG ML and XML publishing applications)

http:/ / www.sdsc.edu/ vrml (The VRML Repository)

http:/ / www.hitl.washington.edu/ projects/ knowledge_base/ vrml_bibliography.html/ (Virtual Reality - VRML)

http:/ / www.intervista.com/ worldview/ (WorldV iew by Intervista Software)

http:/ / www.primate.wisc.edu/ software/ RTF/ D ocuments/ (RTF-Tools documents)

http:/ / www.tug.org/ (TeX Users Group Home Page)

http:/ / www.cl.cam.ac.uk/ TeX doc/ TeX docs.html (TeX-related documentation)

http:/ / www.math.upenn.edu/ TeX.html(TeX)

http:/ / yoyo.cc.monash.edu.au/ wigs/ postscript/ (Internet PostScript Resources)

http:/ / ds.dial.pipex.com/ quite/ psintro.htm (All about PostScript)

http:/ / www.adobe.com/ prodindex/ postscript/ overview.html (Adobe PostScript Overview)

http:/ / grouchy.cs.indiana.edu/ docproject/ programming/ postscript/ postscript.html (A First Guide to PostScript)

http:/ / www.oclc.org/ oclc/ scholar.htm (The New Electronic Scholarship Homepage)

\section{R evistas electrónicas}

http:/ / www.press.umich.edu/ digpub/ jep.html (The University of Michigan Press)

\section{Productos electrónicos/digitales}

http:/ / www.public.iastate.edu/ CYBERSTACKS/ Aristotle.htm 
Project Aristotle(sm): Automated Categorization of Web Resources

\section{Productos digitales}

http:/ / www.whatis.com/ (whatis.com)

http:/ / www.pcwebopaedia.com/ (PC Webopaedia home page)

\section{Ediciones electrónicas-en línea}

http:/ / www.eBLAST.com/ (eBLAST : Encyclopædia Britannica's Intemet Guide)

http:/ / www.eb.com/ (Welcome to Britannica Online)

http:/ / ibis.nott.ac.uk/ guidelines/ index.html (New Frontiers of Learning Contents)

\section{Ediciones electrónicas-C D R OM}

http:/ / ibis.nott.ac.uk/ memb/ (Multimedia Encyclopedia of Mammalian Biology)

http:/ / gme.grolier.com/ (G rolier Multimedia Encyclopedia O nline - Introduction)

\section{Foros de intercambio}

http:/ / www.free-press.com/ (Internet Free-Press)

\section{Biblioteca digital/electrónica/virtual}

http:/ / www.educ.sfu.ca/ gentech/ libweb.htm (Libraries in the Digital Age)

\section{Proyectos}

Se indizan una serie de direcciones sobre proyectos de biblioteca digital http:/ / sunsite.berkeley.edu/ UCD L/ title.html (The UC D igital Library: A

Framework for Planning and Strategic Initiatives)

http:/ / www.bartleby.com/ index.html (The New Bartleby: A National Digital Library)

http:// sailor.gutenberg.org/ (Project Gutenberg at Sailor) 
http:// www.library.ucsf.edu/ (GALEN II - The UCSF Digital Library)

http:/ / www.cecm.sfu.ca/ publications/ publications.html (About the CECM)

http:/ / www.mannlib.comell.edu/ (Mann Library at Cornell University)

http:/ / www.cnri.reston.va.us/ home/ cstr.html (Computer Science Technical Reports)

http:/ / sunsite.berkeley.edu/ Berkeley Digital Library SunSITEA

\section{Recursos}

Se indizan una serie de direcciones sobre biblioteca digital http:/ / info.lib.uh.edu/ sepb/ rdiglib.htm (Resources: Digital Libraries)

http:/ / www.yahoo.com/ Reference/ Libraries/ Digital_Libraries/ (Yahoo! Reference:Libraries:D igital Libraries)

http:/ / www.texshare.edu/ Services/ Professional/ digital.html (Building Digital Libraries)

\section{Bibliotecas virtuales}

http:/ / vib.stanford.edu/ Overview.html (WWW Virtual Library)

\section{Bibliotecas electrónicas}

http:/ / www.elibrary.com/ (Electric Library Personal Edition)

http:/ / www.dgbiblio.unam.mx/ (Web de la Dir. Gral. de Bibliotecas, UNAM)

\section{B uscadores de revistas electrónicas}

http:/ / web.usal.es/ eus/ recursos/ rev-e.html (RED ERO, Angel Luis. "Buscadores de revistas electrónicas)

\section{Máquinas de búsqueda}

http:/ / imt.net/ notess/ search/ index.html (Search Engines Showdown)

http:/ / www.altavista.digital.com/ (AltaVista: Main Page)

http:// www.excite.com/ (Excite)

http:// www.northernlight.com/ (Northem Light Search)

http:/ / www.infoseek.com/ (Infoseek)

\section{Directorios}


http:/ / www.looksmart.com/ (LookSmart - exploring World)

http:// www.yahoo.com/ (Yahoo)

\section{Máquinas multi- búsqueda}

Realiza multiples búsquedas paralelas en bases de datos de Web http:/ / profusion.ittc.ukans.edu/ (ProFusion)

http:/ / www.metacrawler.com/ (MetaCrawler)

http:/ / guaraldi.cs.colostate.edu:2000/ (SavvySearch)

http:/ / www.metafind.com/ (MetaFind Your Search Spot on the Net)

http:/ / www.dogpile.com/ (Welcome to D ogpile, the Friendly Multi-Engine Search Tool)

\section{A rtículos \\ revistas académicas}

http:/ / www.info.unicaen.fr/ bnum/ jelec/ Solaris/ (Solaris: revue électronique française/ french electronic journal)

http:/ / www.urfist.jussieu.fr/ urfist/ revues.htm (Revues électroniques sur les réseaux)

http:/ / www.oclc.org/ oclc/ research/ publications/ weibel/ web_pub_arch/web_pub_arch.html (An Architecture for Scholarly Publishing on the World Wide Web)

http:/ / www.info.unicaen.fr/ bnum/ jelec/ Solaris/ d03/ 3lecrosnier.html (Les joumaux scientifiques électroniques ou la communication de la science à l'heure du réseau mondial)

http:/ / www.info.unicaen.fr/ bnum/ jelec/ Solaris/ d03/ 3chartron.html (La presse périodique scientifique sur les réseaux)

http:/ / www.soi.city.ac.uk/ course/ msc/ elpub/ 1.html (The School of Informatics, City University)

\section{V isualizadores}

http:/ / browserwatch.internet.com/ (BrowserWatch Home Page)

\section{Conferencias}

http:/ / www.gpd.org/ maig98/ (Maig ‘98, I Congrés de la Publicació Digital) http:/ / www.lmcp.jussieu.fr/ icsu/ Information/ Proc_0296/ index.html (Electronic Publishing in Science)

\section{Directrices}

http:/ / sunsite.berkeley.edu/ MLA/ guidelines.html (Guidelines for Electronic Scholarly Editions) 
http:/ / sunsite.berkeley.edu/ MLA/ principles.html (General Principles for Electronic Scholarly Editions)

http:/ / www.alcs.co.uk/ guidelin.htm (ALCS Guidelines for Writers in Electronic Publishing and Multimedia)

\section{Publicación digital-proyecto}

http:/ / www-mitpress.mit.edu/ electronic.html (Electronic Publications)

http:/ / www.press.umich.edu/ digpub/ digpub.html (The University of Michigan Press)

\section{A sociaciones-editoras}

$\mathrm{http/} /$ uwwasisarg (Ammican Socidy for Infomation SaieneHomePage)

\section{Programas de autor}

http:// www.adobe.com/ (Adobe Systems Incorporated)

\section{Estudios-economía}

http:/ / www2.echo.lu/ impact/ projects/ studies/ en/ electrpub.html (Strategic Study on Electronic Publishing)

\section{D irectorio sobre publicación electrónica}

http:/ / scholar.lib.vt.edu (Scholarly Communications Project, University Libraries, Virginia Tech)

http:/ / www.sil.org/ general/ epub.html (Electronic Publishing)

http:/ / acm.org/ SIG LINK/ ECHT94TR/ O SISN.html ((O pen Systems, Information Structuring, and Navigation) 